\title{
Sagittal component alignment is less reliable than coronal component alignment in a Chinese population undergoing navigated TKA
}

Xiaoyong Chen ${ }^{1,2}$, Huayi Wang ${ }^{1}$, Yuanzhen Cai ${ }^{1}$, Qingsheng Zhu ${ }^{1 *+}$ and Jinyu Zhu ${ }^{1 *+}$

\begin{abstract}
Background: The purpose of our study was to determine whether postoperative sagittal component alignments of primary total knee arthroplasty (TKA) using the conventional and navigated technique differed significantly. Additionally, we determined whether the use of navigation systems resulted in hyperextension of the femoral components in Chinese patients.

Methods: This retrospective study reviewed 36 consecutive patients (72 knees) who underwent simultaneous bilateral primary TKAs at our hospital from February 2011 to March 2012. One knee was replaced using a computer-assisted navigation system, and the contralateral knee was replaced with the conventional technique. The radiographic and clinical results of both groups were compared. The relationship between preoperative anatomic angles and component alignments in conventional TKA and navigated TKA was examined.

Results: The radiographic results showed statistically significant differences only between the navigated and conventional groups for individual femoral coronal and sagittal component alignment. Femoral sagittal component alignment showed less deviation and tended to have hyperextension using the navigated technique $\left(-0.35^{\circ}\right)$ compared with the conventional technique $\left(2.77^{\circ}\right)$. There was no significant difference observed for the Knee Society Score (KSS) between the two groups at 2 years postoperatively.

Conclusions: The sagittal component alignment of primary TKA obtained using the conventional and navigated techniques differed significantly. Navigated TKAs resulted in a higher risk of hyperextension of the femoral components in Chinese patients.
\end{abstract}

Keywords: Total knee arthroplasty, Computer-assisted, Sagittal alignment, Navigation

\section{Background}

Computer navigation systems are designed to increase the accuracy and consistency of prosthetic alignment in total knee arthroplasty (TKA). Many studies have shown a reduction in the number of outliers, that is, misalignments of $>3^{\circ}$, in both the components and the lower limb mechanical axis using the navigated technique [1-6]. The accuracy of prosthetic alignment in the sagittal plane has been less emphasised in previous studies compared to coronal component alignment.

\footnotetext{
* Correspondence: zhuqingsheng11@live.com; xjhospitalzjy@gmail.com

${ }^{\dagger}$ Equal contributors

'Department of Orthopedics, Xijing Hospital, Fourth Military Medical University, Xi'an 710032, China

Full list of author information is available at the end of the article
}

Sagittal alignment of the femoral component may influence the clinical results of TKA in various ways. If a femoral component is placed in hyperflexion, the extension or polyethylene post wear resulting from impingement between the anterior part of the polyethylene insert and the intercondylar box of the femoral component in TKA can be limited [7]. When a femoral component is placed in hyperextension relative to the femur, it may create a notch in the anterior femoral cortex, which can increase the potential risk of a supracondylar fracture $[8,9]$. Based on these data, ignorance of sagittal component alignment in TKA is not acceptable. A previous study examining the use of standing radiographs of the entire lower extremity has shown that targeted sagittal component alignments of TKA achieved using 
the conventional and navigated techniques differed significantly. The use of navigation systems resulted in hyperextension between the femoral and tibial components [10]. However, the subjects included in the previous study were healthy volunteers without symptoms or radiographic abnormalities. Thus, these results may not be directly applicable to patients with lower limb deformity. In the Chinese population, bowing of the femur is commonly found in patients with osteoarthritis of the knee. The incidence of femur bowing is approximately $62 \%[11,12]$.

The purpose of our study was to determine whether postoperative sagittal component alignments of simultaneous bilateral primary TKAs obtained using the conventional and navigated techniques differed significantly and whether the use of navigation systems resulted in hyperextension of the femoral components in Chinese patients undergoing navigated TKAs.

\section{Patients and methods Demographics}

We retrospectively reviewed the hospital records of 40 consecutive patients (80 knees) who underwent simultaneous bilateral primary TKAs at our hospital from February 2011 to March 2012. One knee was replaced using a computer-assisted navigation system. The contralateral knee was replaced with a conventional technique using an intramedullar rod for the femur and a mechanical extramedullar guiding system for the tibia. The indications for surgery were rheumatoid arthritis and knee osteoarthritis. There were two patients with bilateral valgus knees and one patient with a unilateral valgus knee, who were excluded from the analysis. Furthermore, one (2.5\%) patient was lost during the follow-up. In total, 36 patients with 72 TKAs were successfully followed up for more than 24 months (Table 1).

There were 30 female and 6 male patients. The sex distribution of arthritis is a common finding in a Chinese ethnic group. There were no significant differences in the

Table 1 Patient demographics

\begin{tabular}{ll}
\hline & Total \\
\hline Number of patients & 40 \\
Gender & \\
$\quad$ Male & 6 \\
$\quad$ Female & 30 \\
Aetiology & 2 \\
$\quad$ Rheumatoid arthritis & 34 \\
$\quad$ Osteoarthritis & $25.2 \pm 4.6$ \\
BMI $^{\text {a }}$ (kg/m ${ }^{2}$ ) & $61.3 \pm 9.5$ \\
Age $^{\text {a }}$ (years) &
\end{tabular}

${ }^{a}$ The values are given as the mean and the standard deviation. preoperative anatomic angles and Knee Society Score (KSS) (Table 2).

\section{Surgical techniques}

All knees were implanted with NexGen posterior-stabilised total knee prosthesis (Zimmer, Warsaw, Indiana). The Stryker Precision Knee navigation-assisted system (Stryker-Leibinger, Freiburg, Germany) was employed. All surgeries were performed by two surgeons (QZ and JZ) experienced in using the Zimmer NexGen prosthesis. The procedure was performed through a midline skin incision of 10 to $12 \mathrm{~cm}$ in length with the use of a medial parapatellar arthrotomy. In the navigated cohort, the surgeon dialled for correct orientation of the jig according to the data presented by the navigation system. Once in position, the surgeon assured a stabile cut block fixation by tightening with pins. All resections were performed manually and verified by the universal tracker. In the conventional cohort, extramedullary instrumentation was used for the tibial component, and intramedullary instrumentation was used for the femoral side. After the bone resection in all knees, the contracted amount of soft tissue was carefully evaluated, and selective release was performed as required. The same protocol for postoperative management was utilised in both groups, which included bedside continuous passive motion machine therapy, physical therapy with partial weight bearing, and quadriceps and hamstring strengthening exercises starting on the second postoperative day.

\section{Radiological evaluation}

Standard anteroposterior and lateral long-leg standing $\mathrm{X}$-rays were obtained before and 6 weeks after surgery to determine the following parameters: the coronal lowerlimb mechanical axis angle (MA) between the coronal femoral mechanical axis and the coronal tibial mechanical axis, the femoral anatomic valgus angle (FAV) between the coronal femoral mechanical axis and the distal femoral anatomic axis, and the femoral anatomic flexion angle (FAF) between the sagittal femoral mechanical axis and the femoral anatomic axis. As described by $\mathrm{Hsu}$ et al. $[13,14]$, the component alignments were evaluated by measuring four modified angles, i.e., (1) the coronal

\section{Table 2 Preoperative radiographical and clinical} measurements

\begin{tabular}{llll}
\hline & Navigation & Convention & $\boldsymbol{p}$ value \\
\hline Mechanical axis & $9.69^{\circ} \pm 5.49^{\circ}$ & $9.45^{\circ} \pm 5.20^{\circ}$ & 0.620 \\
Femoral anatomic valgus & $6.56^{\circ} \pm 2.68^{\circ}$ & $6.66^{\circ} \pm 2.77^{\circ}$ & 0.627 \\
Femoral anatomic flexion & $4.03^{\circ} \pm 1.44^{\circ}$ & $4.15^{\circ} \pm 1.51^{\circ}$ & 0.336 \\
Knee score & $34.97 \pm 10.16$ & $34.36 \pm 10.17$ & 0.063 \\
Function score & $45.28 \pm 11.08$ & $44.03 \pm 12.06$ & 0.141 \\
\hline
\end{tabular}

There was no significant difference between two groups $(p>0.05)$. 
femoral angle $(\mathrm{CF})$ between the mechanical axis of the femur and the transcondylar line of the femur as measured on the medial side, (2) the coronal tibial angle (CT) between the mechanical axis of the tibia and the tibial base plate as measured on the medial side, (3) the sagittal femoral angle (SF) between the sagittal femoral mechanical axis and the perpendicular axis of the femoral component and (4) the sagittal tibial angle (ST) between the sagittal tibial mechanical axis and the horizontal axis of the tibial component (Figure 1). The target values for implantation were recommended by the manufacturer: a MA of $0^{\circ}$, a CF of $90^{\circ}$ for femoral component and a CT of $90^{\circ}$ for the tibial component in the coronal plane. In the sagittal plane, the target value was an SF of $0^{\circ}$ for the femoral component and a ST of $85^{\circ}$ for the tibial component. The values of the mechanical axes with varus deformity were recorded as positive values. The goal of TKA implantation was to achieve a postoperative correction in the mechanical axis within a range of $3^{\circ}$ varus or valgus. A radiological evaluation was performed by two independent observers ( $\mathrm{YC}$ and $\mathrm{HW}$ ). The results are expressed as the mean, range and standard deviation.

\section{Clinical evaluation}

All patients were routinely assessed before surgery and then 6 months and annually after surgery. The KSS score was used for clinical evaluation $[15,16]$. The KSS score is divided into the knee score and the function score. The patients underwent clinical evaluation at the outpatient clinic. Whenever it was not possible to come to the outpatient clinic (mainly because of transportation problems), these patients were visited by the examiner of a local hospital and were interviewed by telephone questionnaire. This was in agreement with the literature that supported the quality of data obtained using the telephone method [16].

\section{Statistical analysis}

Statistical analyses were performed using SPSS 20 (SPSS Inc., Chicago, IL, USA) software. The preoperative data of both groups were compared using Student's $t$ test. The radiographic and clinical results of both groups were compared using Student's $t$ test and chi-square test. The relationship between preoperative anatomic angles and component alignments in conventional TKA and navigated TKA was examined using the Spearman correlation coefficient. The $p$ value $<0.05$ indicates a statistically significant difference.

\section{Results}

All patients were followed up for an average of 30.5 months (range, 24-36 months). There was no significant difference in postoperative complications between the two groups. There was one case of deep vein thrombosis in the navigated group and one case of serious bruises in the conventional group. One patient with bilateral knees required manipulation under anaesthesia because of knee stiffness. There were no patients who required additional surgery due to implant failure during the follow-up period. Surgery using the navigated technique lasted an average of $92 \mathrm{~min}$ for a TKA. Surgery using the conventional technique lasted an average of $60 \mathrm{~min}(p<0.001)$.

The postoperative lower-limb alignment demonstrated more varus in the conventional group $\left(2.25^{\circ} \pm 3.14^{\circ}\right)$ than in the navigated group $\left(1.19^{\circ} \pm 1.56^{\circ}\right) \quad(p=0.028)$. There was a significant difference between these two measures

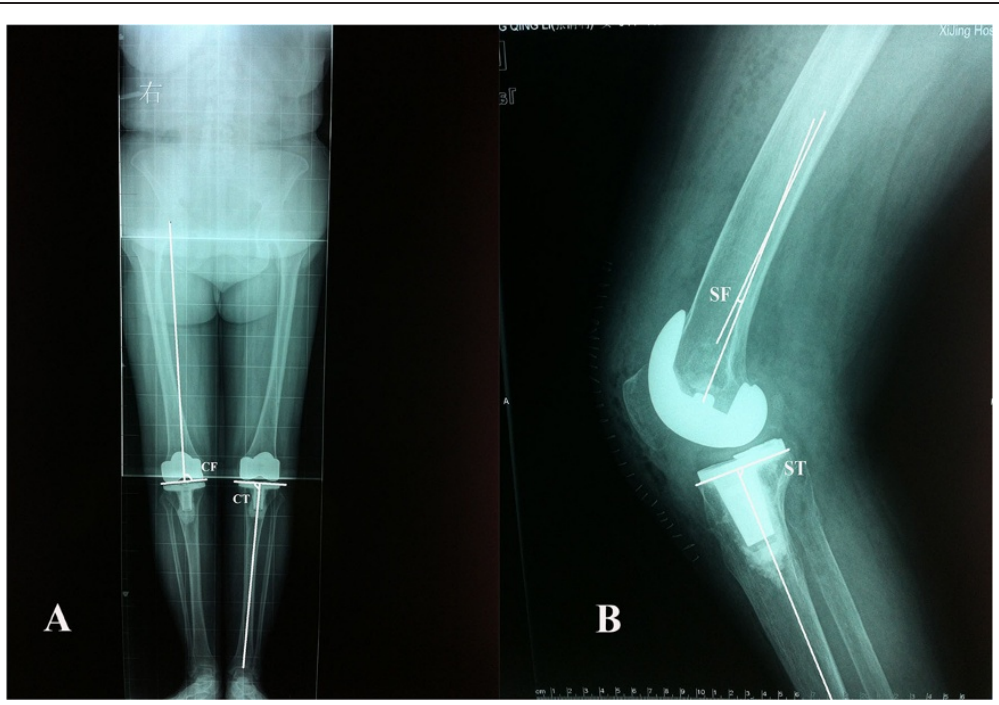

Figure 1 Measurement of various angles on anteroposterior (A) and lateral (B) radiographs after total knee arthroplasty. The component alignments were evaluated by measuring four modified angles including the coronal femoral angle (CF), coronal tibial angle (CT), sagittal femoral angle (SF) and sagittal tibial angle (ST). 
with regard to the number of knees outside $\pm 3^{\circ}$ of a neutral mechanical axis $(p=0.032)$. The navigated group had $8.3 \%(3 / 36)$ of knees outside $\pm 3^{\circ}$ of neutral, whereas the conventional group had $27.8 \%$ (10/36). Additional alignment data can be found in Table 3. The femoral components in the conventional group $\left(89.25^{\circ} \pm 3.32^{\circ}\right)$ tended to be more varus than in the navigated group $\left(90.60^{\circ} \pm 1.75^{\circ}\right)$ $(p=0.014)$. In the sagittal plane, the femoral components inserted with navigation tended toward hyperextension $\left(-0.35^{\circ} \pm 1.45^{\circ}\right)$ vs. flexion $\left(2.77^{\circ} \pm 2.21^{\circ}\right)$ in the conventional group $(p<0.001)$. Similarly, the femoral components in the navigated group demonstrated less deviation from the femoral mechanical axis $(p=0.003)$. Simultaneously, the tibial component alignment tended to be in slight varus and flexion in both groups, and there was no significant difference.

There was a correlation $(r=-0.754, p<0.001)$ between the femoral anatomic valgus angle and postoperative coronal femoral angle in the conventional group. However, no correlation was found in the navigated patients. Similarly, there was a positive correlation $(r=0.836, p<0.001)$ demonstrated between the femoral anatomic flexion and postoperative sagittal femoral angle in the conventional group. There was no correlation found in the navigated group (Figure 1).

The KSS scores are shown in Table 4. The knee score and function score were not different between the navigated and conventional groups $(73.83 \pm 9.84$ vs. $75.14 \pm$ 9.74 and $73.89 \pm 10.69$ vs. $72.78 \pm 11.18$ ) at 6 months postoperatively. There was no significant difference of KSS between the two groups at 2 years postoperatively.

\section{Discussion}

The most important finding of the present study was that postoperative sagittal component alignments of simultaneous bilateral primary TKA obtained using the conventional and navigated techniques differed significantly. Notably, the use of navigation systems resulted in hyperextension of the femoral components in Chinese patients undergoing TKAs.

Previous randomised control trials comparing conventional total knee arthroplasty with computer-assisted surgery have demonstrated a smaller range of deviation from all component alignments and fewer outliers with computer-assisted surgery [17-22]. However, other studies have failed to show a significant difference [23,24]. In this comparative study, there were no significant differences in most variables for patients who underwent simultaneous bilateral primary TKAs. The evaluations of the radiographic results showed statistically significant differences only between the navigated and conventional groups for the femoral component. The overall percentage of TKAs that achieved the target component alignment in the femoral valgus and flexion angles was also higher with the navigated technique.

In our study, postoperative femoral component alignment in the sagittal plane using the conventional and navigated techniques differed significantly. However, it was inconclusive whether sagittal component alignment after navigated surgery was significantly better than the conventional technique. The sagittal femoral component alignment showed less deviation and tended to be in hyperextension using the navigated technique compared with the conventional technique. The angles were $-0.35^{\circ}$ in the navigated group and $2.77^{\circ}$ in the conventional group. Femoral component flexion allows for impingement of the femoral cam on the anterior aspect of the tibial post leading to anterior post wear and deformation, which can cause an increase in rotational constraint in knee extension. The hyperextension position of the femoral components increases the potential risk of osteolysis and anterior tibial post impingement with posterior stabilised prostheses [25]. Therefore, sagittal femoral component alignment in navigated TKA is less reliable than coronal component alignment. Although this parameter has not often been described in prior studies, the outcome was not unpredictable because the navigated technique does not account for the anterior bow of the femur. The centre of the femoral head and the distal femoral inputs tend to extend the femoral position. However, femoral bowing changes the angular relationship between the anatomical axis and the mechanical axis of the distal femur. This relationship determines the choice of the femoral cutting block and the distal femoral bone cut when using the conventional technique (Figure 2). If an intramedullary guide rod is inserted, the rod will follow the alignment

Table 3 Postoperative radiographical measurements and outliers of lower-limb and component alignments

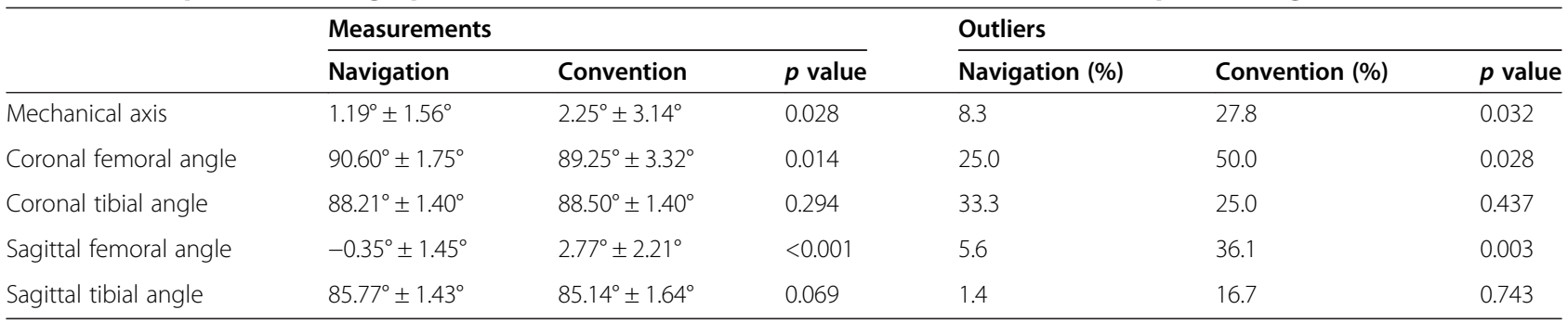

An average value \pm standard deviation is reported for each index. 
Table 4 Postoperative clinical results at 6 months and 2 years

\begin{tabular}{|c|c|c|c|c|c|c|}
\hline & \multicolumn{3}{|l|}{6 months } & \multicolumn{3}{|l|}{2 years } \\
\hline & Navigation & Convention & $p$ value & Navigation & Convention & $p$ value \\
\hline Knee score & $73.83 \pm 9.84$ & $75.14 \pm 9.74$ & 0.285 & $84.89 \pm 7.85$ & $85.28 \pm 8.39$ & 0.224 \\
\hline Function score & $73.89 \pm 10.69$ & $72.78 \pm 11.18$ & 0.210 & $83.06 \pm 9.43$ & $82.08 \pm 10.31$ & 0.255 \\
\hline
\end{tabular}

An average value \pm standard deviation is reported for each index.

of the distal femur. Because of the femoral bowing, conventional technique can never make the longitudinal axes of the implant and the femur parallel to each other. We recommend that it is important to modify the flexion angle of the femoral component suggested by the navigation software according to the femoral bowing. Anatomically, registration using an imageless bone morphing technique requires precise and widespread contact with the anterior femoral cortex by the navigation pointer to achieve sufficient information about distal femoral flexion and curvature. However, there is typically a significant amount of soft tissue on the anterior femur, and bone morphing may be impaired or inaccurate. We suggest that this is the source of the relative inaccuracy in the navigated knees.

Although this study reported no significant difference with tibial component alignments between navigated surgery and conventional surgery, there was a trend toward lower percentages of patients with misalignment outside of the acceptable range after navigated surgery. We suggest that the tibial coronal cut is a critical step. All subsequent femoral cuts and soft tissue balancing are based on the accuracy of this initial step. Furthermore, tibial loosening appears to be the most frequent fixation failure leading to early revision surgery [26-29]. Accordingly, the superior results associated with navigation relative to the tibial coronal cut are important to note.

Although the overall percentage of TKAs that achieved the target component alignments was higher with the navigated technique, there were no differences in postoperative KSS scores. There was no clear short-term clinical benefit to navigated TKA identified in this study. There may be additional technical factors besides lowerlimb mechanical alignment contributing to the pattern of outcomes noted. These factors include soft tissue balance and joint line location [30], which are not only difficult to assess but challenging to accurately quantify. A dynamic
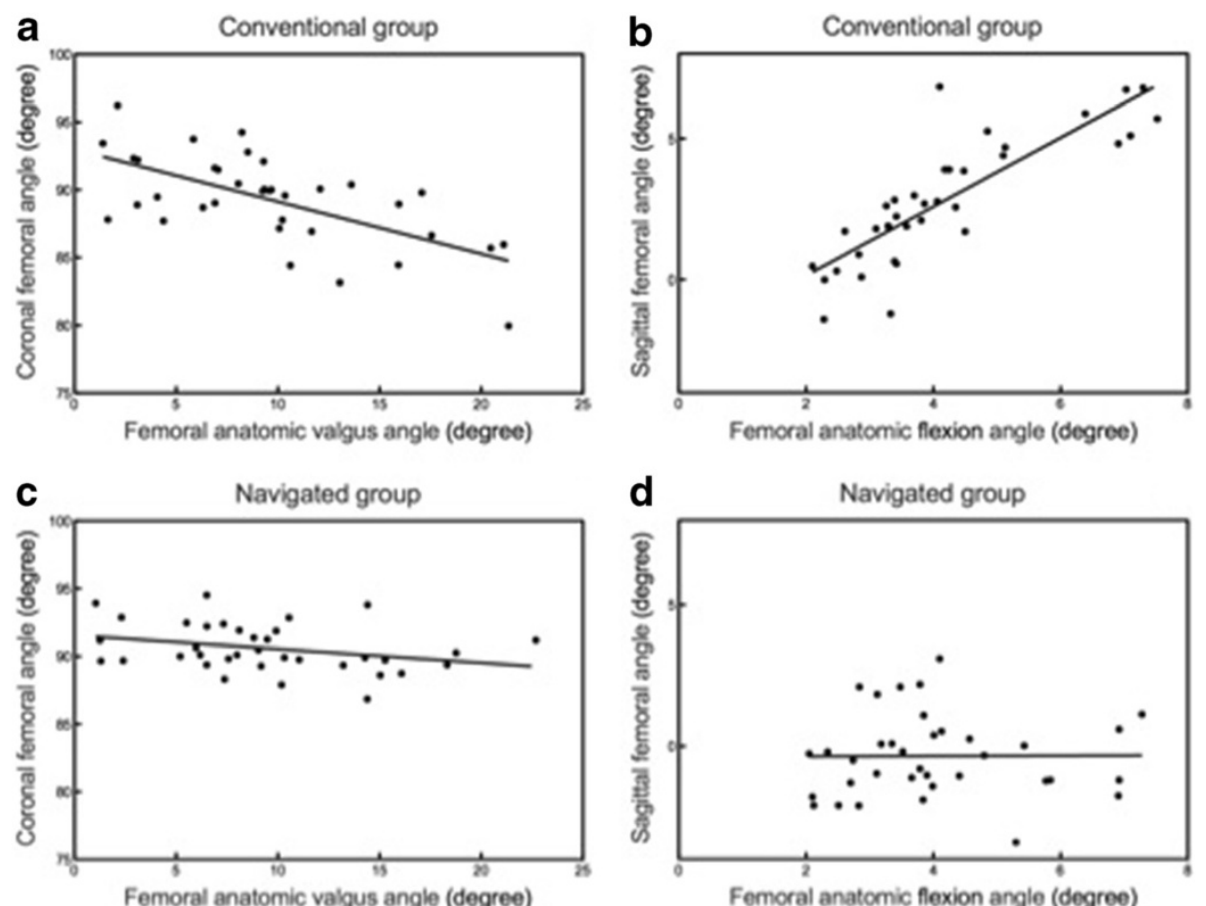

Figure 2 Scatter plot showing a linear regression analysis of conventional group $(\mathbf{a}, \mathbf{b})$ and navigated group (c, d). The preoperative femoral anatomic angle influenced the postoperative femoral component angle in conventional TKA, whereas no such correlation was found in the navigated patients. 
gait pattern is also emerging as a possible important driver of outcome after TKA. Therefore, postoperative functions of TKAs are determined by multiple factors. There is still a potential risk that the importance of the mechanical alignment of the lower limb is overemphasised, and the role of other factors is ignored.

Several limitations in this study should be acknowledged. We did not randomise our patients. However, to limit selection bias, we reviewed consecutive patients who underwent simultaneous bilateral primary TKAs, which eliminated or reduced the effects of confounders. Additionally, it was relatively accurate to evaluate postoperative pain score by comparing bilateral knee joints for every patient. Another potential bias of the study is that the use of navigated or conventional TKA on the first knee may have provided information in planning for the contralateral knee. Second, a major limitation is that we did not use three-dimensional CT scans, which may have revealed differing results with regard to the radiographic outcomes assessed such as rotational positions of the femoral and tibial components. However, there were no intraoperative or postoperative patella complications and no insufficiencies of the knee extensor mechanism. In addition, it is difficult to perform CT while a subject is in the standing position. Third, the study lacked blinding of the radiographic review. It is difficult to perform a blinded review because the radiographic features of the pin tracts for the navigation arrays are often present.

\section{Conclusions}

In summary, our retrospective, self-control study showed postoperative sagittal component alignments of simultaneous bilateral primary TKA obtained using the conventional and navigated techniques differed significantly. Using the navigated technique resulted in a higher risk of hyperextension of the femoral components compared to the conventional technique in Chinese patients undergoing TKAs.

\section{Competing interests}

The authors declare that they have no competing interests.

\section{Authors' contributions}

$\mathrm{XC}$ participated in the design of the study and drafted the manuscript. JZ and QZ participated in the design of the study and coordination and helped draft the manuscript. YC and HW participated in the radiological evaluation and performed the statistical analysis. All authors read and approved the final manuscript.

\section{Acknowledgements}

The authors would like to thank Dr. Kai Guo for his help and support in the statistical analysis. We are also grateful to Dr. Bo Ren for editing this manuscript.

\section{Author details}

'Department of Orthopedics, Xijing Hospital, Fourth Military Medical University, Xi'an 710032, China. ${ }^{2}$ Department of Orthopedics, Chenggong Hospital affiliated to Xiamen University, Xiamen 361000, China.
Received: 27 March 2014 Accepted: 16 June 2014

Published online: 06 July 2014

\section{References}

1. Ong A, Jung KA, Orozco F, Delasotta L, Lee DW: Total knee arthroplasty using a hybrid navigation technique. J Orthop Surg Res 2011, 6:26.

2. Zhang GQ, Chen JY, Chai W, Liu M, Wang Y: Comparison between computer-assisted-navigation and conventional total knee arthroplasties in patients undergoing simultaneous bilateral procedures: a randomized clinical trial. J Bone Joint Surg Am 2011, 93:1190-1196.

3. Huang TW, Hsu WH, Peng KT, Hsu RW, Weng YJ, Shen WJ: Total knee arthroplasty with use of computer-assisted navigation compared with conventional guiding systems in the same patient: radiographic results in Asian patients. J Bone Joint Surg Am 2011, 93:1197-1202.

4. Molli RG, Anderson KC, Buehler KC, Markel DC: Computer-assisted navigation software advancements improve the accuracy of total knee arthroplasty. J Arthroplast 2011, 26:432-438.

5. Mason JB, Fehring TK, Estok R, Banel D, Fahrbach K: Meta-analysis of alignment outcomes in computer-assisted total knee arthroplasty surgery. J Arthroplast 2007, 22:1097-1106.

6. Chin PL, Yang KY, Yeo SJ, Lo NN: Randomized control trial comparing radiographic total knee arthroplasty implant placement using computer navigation versus conventional technique. J Arthroplast 2005, 20:618-626.

7. Stoller AP, Johnson TS, Popoola OO, Humphrey SM, Blanchard CR: Highly crosslinked polyethylene in posterior-stabilized total knee arthroplasty: in vitro performance evaluation of wear, delamination, and tibial post durability. J Arthroplast 2011, 26:483-491.

8. Li G, Papannagari R, Most E, Park SE, Johnson T, Tanamal L, Rubash HE: Anterior tibial post impingement in a posterior stabilized total knee arthroplasty. J Orthop Res 2005, 23:536-541.

9. Haas BD: Tibial post impingement in posterior-stabilized total knee arthroplasty. Orthopedics 2006, 29:S83-S85.

10. Minoda Y, Kobayashi A, Iwaki H, Ohashi H, Takaoka K: TKA sagittal alignment with navigation systems and conventional techniques vary only a few degrees. Clin Orthop Relat Res 2009, 467:1000-1006.

11. Yau W, Chiu K, Tang W, Ng T: Coronal bowing of the femur and tibia in Chinese: its incidence and effects on total knee arthroplasty planning. J Orthop Surg 2007, 15:32-36

12. Mullaji AB, Marawar SV, Mittal V: A comparison of coronal plane axial femoral relationships in Asian patients with varus osteoarthritic knees and healthy knees. J Arthroplast 2009, 24:861-867.

13. EWALD FC: The knee society total knee arthroplasty roentgenographic evaluation and scoring system. Clin Orthop Relat Res 1989, 248:9-12.

14. Hsu RW, Himeno S, Coventry MB, Chao EY: Normal axial alignment of the lower extremity and load-bearing distribution at the knee. Clin Orthop Relat Res 1990, 255:215-227.

15. Marx RG: Knee rating scales. Arthroscopy 2003, 19:1103-1108

16. McGrory B, Shinar A, Freiberg A, Harris W: Enhancement of the value of hip questionnaires by telephone follow-up evaluation. J Arthroplast 1997, 12:340-343.

17. Gregori A, Holt G: Navigated total knee replacement. A meta-analysis. J Bone Joint Surg 2007, 89:1867-1867.

18. Dutton AQ, Yeo S-J, Yang K-Y, Lo N-N, Chia K-U, Chong H-C: Computer-assisted minimally invasive total knee arthroplasty compared with standard total knee arthroplasty. A prospective, randomized study. J Bone Joint Surg 2008, 90:2-9.

19. Matziolis G, Krocker D, Weiss U, Tohtz S, Perka C: A prospective, randomized study of computer-assisted and conventional total knee arthroplasty. Three-dimensional evaluation of implant alignment and rotation. J Bone Joint Surg 2007, 89:236-243.

20. Chauhan S, Scott R, Breidahl W, Beaver R: Computer-assisted knee arthroplasty versus a conventional jig-based technique. A randomised, prospective trial. J Bone Joint Surg, British Volume 2004, 86:372-377.

21. Decking R, Markmann Y, Fuchs J, Puhl W, Scharf H-P: Leg axis after computer-navigated total knee arthroplasty: a prospective randomized trial comparing computer-navigated and manual implantation. J Arthroplast 2005, 20:282-288.

22. Choong PF, Dowsey MM, Stoney JD: Does accurate anatomical alignment result in better function and quality of life? Comparing conventional and computer-assisted total knee arthroplasty. J Arthroplast 2009, 24:560-569. 
23. Lützner J, Krummenauer F, Wolf C, Günther K-P, Kirschner S: Computer-assisted and conventional total knee replacement: a comparative, prospective, randomised study with radiological and $\mathrm{CT}$ evaluation. J Bone Joint Surg, British Volume 2008, 90:1039-1044.

24. Chauhan S, Clark G, Lloyd S, Scott R, Breidahl W, Sikorski J: Computer-assisted total knee replacement. A controlled cadaver study using a multi-parameter quantitative CT assessment of alignment (the Perth CT Protocol). J Bone Joint Surg, British Volume 2004, 86:818-823.

25. Callaghan JJ, O'Rourke MR, Goetz DD, Schmalzried TP, Campbell PA, Johnston RC: Tibial post impingement in posterior-stabilized total knee arthroplasty. Clin Orthop Relat Res 2002, 404:83-88.

26. Goldberg VM, Kraay M: The outcome of the cementless tibial component: a minimum 14-year clinical evaluation. Clin Orthop Relat Res 2004, 428:214-220.

27. Barrack RL, Nakamura SJ, Hopkins SG, Rosenzweig S: Winner of the 2003 James A. Rand young investigator's award: early failure of cementless mobile-bearing total knee arthroplasty. J Arthroplast 2004, 19:101-106.

28. Peters CL, Erickson J, Kloepper RG, Mohr RA: Revision total knee arthroplasty with modular components inserted with metaphyseal cement and stems without cement. J Arthroplast 2005, 20:302-308.

29. Solomon LB, Stamenkov RB, MacDonald AJ, Yaikwavong N, Neale SD, Moss MJ, Howie DW: Imaging periprosthetic osteolysis around total knee arthroplasties using a human cadaver model. J Arthroplast 2012, 27:1069-1074.

30. Sikorski J: Alignment in total knee replacement. J Bone Joint Surg, British Volume 2008, 90:1121-1127.

doi:10.1186/s13018-014-0051-

Cite this article as: Chen et al: Sagittal component alignment is less reliable than coronal component alignment in a Chinese population undergoing navigated TKA. Journal of Orthopaedic Surgery and Research 2014 9:51.

\section{Submit your next manuscript to BioMed Central and take full advantage of:}

- Convenient online submission

- Thorough peer review

- No space constraints or color figure charges

- Immediate publication on acceptance

- Inclusion in PubMed, CAS, Scopus and Google Scholar

- Research which is freely available for redistribution 